\title{
BUILDINGS AS INNER IDEAL GEOMETRIES
}

\author{
MEIGHAN I. DILLON
}

(Communicated by Lance W. Small)

\begin{abstract}
We consider a Chevalley-type group $G(V)$ associated to an integrable representation of a Kac-Moody algebra and show that the associated KacMoody group $G(A)$ is a universal cover for $G(V)$. This observation strengthens a result of Kac-Peterson on representations of $G(A)$. It also implies that the building associated to an affine Lie algebra can be realized as an inner ideal geometry.
\end{abstract}

We refer the reader to [4] for details concerning Kac-Moody algebras and their representations.

Let $S$ be a finite index set, and let $\mathbf{g}$ be the Kac-Moody algebra associated to a generalized Cartan matrix, $A=\left(a_{i j}\right)_{i, j \in S}$. We assume throughout that $A$ is indecomposable.

Let $\mathbf{h}$ be a Cartan subalgebra for $\mathbf{g}$. Its linear dual is $\mathbf{h}^{*}$. Use $\Pi$ to designate the set of simple roots, $\Delta$ the set of roots, $\Delta^{r e}$ the real roots, and $\Delta_{+}^{r e}$ the positive real roots of $\mathbf{g}$. Let $\mathbf{g}=\mathbf{h} \oplus_{\alpha \in \Delta} \mathbf{g}_{\alpha}$ be its root space decomposition. Fix a set of Chevalley generators for $\mathbf{g},\left\{e_{i}, f_{i}: i \in S\right\}$ where $e_{i} \in \mathbf{g}_{\alpha_{i}}$ and $f_{i} \in \mathbf{g}_{-\alpha_{i}}$ for $\alpha_{i} \in \Pi$. When $\mathbf{g}$ is affine, we use $(\cdot, \cdot)$ to designate a standard invariant form.

Kac-Moody groups and buildings. We consider two types of groups associated to $\mathbf{g}$ and its representations. The first is defined in terms of $A$ without reference to $\mathbf{g}$ at all. This is the Kac-Moody group which we designate $G(A)$. It is constructed directly as follows. (This definition is from [8]. See [5] for an axiomatic treatment.)

Let $G^{*}$ be the free product of the additive groups $\mathbf{g}_{\alpha}$ for $\alpha \in \Delta^{r e}$. Denote the canonical injections by $l_{\alpha}: \mathbf{g}_{\alpha} \rightarrow G^{*}$. If $(V, d \pi)$ is an integrable representation of $\mathbf{g}$, then we have a representation $\pi^{*}: G^{*} \rightarrow \operatorname{Aut}_{\mathbf{C}}(V)$ defined by $\pi^{*}\left(l_{\alpha}\left(x_{\alpha}\right)\right)=\exp d \pi\left(x_{\alpha}\right)$. (Since $x_{\alpha}$ is locally nilpotent, $\exp d \pi\left(x_{\alpha}\right)$ is a well-defined automorphism of $V$.) Define $G(A)=G^{*} / N^{*}$ for $N^{*}=\cap \operatorname{ker} \pi^{*}$ where the intersection is taken over all integrable representations of $\mathbf{g}$.

As noted in [5], there is an injective homomorphism $\varphi_{i}: S L(2, \mathbf{C}) \rightarrow G(A)$ for each $i \in S$. The set of all $G_{i}=\varphi_{i}(S L(2, \mathrm{C}))$ generates $G(A)$.

Induce a topology on $G(A)$ by decreeing that the maps $\varphi_{i}$ be continuous with respect to the usual topology on $S L(2, \mathrm{C})$. The Kac-Moody group is then

Received by the editors March 12, 1993.

1991 Mathematics Subject Classification. Primary 17B67, $20 \mathrm{E} 42$.

This research was supported in part by NSF Grant No. DMS-9009268. 
a connected, simply connected topological group which satisfies the Hausdorff separation axiom (cf. [6]).

It is well known [5-8] that $G(A)$ has a $B N$-pair structure (also called a Tits system; cf. [1, Chapter IV, §2.1]). We take our $B N$-pair for $G(A)$ to be as given in [8]. Here we write $B=B(A)$ and $N=N(A)$. Note that when $b \in B(A)$ and $V$ is an integrable highest weight representation of $\mathbf{g}$ with weight space $V_{\lambda}, b \cdot V_{\lambda} \subseteq \sum V_{\rho}$ where $\rho \geq \lambda$. In other words, $\rho-\lambda=\sum_{\alpha_{i} \in \Pi} k_{i} \alpha_{i}$ where $k_{i} \geq 0$ for each $i$.

Fix an integrable representation of $\mathbf{g}, d \pi: \mathbf{g} \rightarrow \operatorname{End}_{\mathbf{C}}(V)$. Let $\pi: G(A) \rightarrow$ $\operatorname{Aut}_{\mathbf{C}}(V)$ be given by $\pi(g)=\pi^{*}\left(g^{*}\right)$ for $g=g^{*} N^{*} \in G(A), g^{*} \in G^{*}$. The second group we consider is $G(V)$, the homomorphic image of $G(A)$ under $\pi$. With the quotient topology, $G(V)$ is a connected topological group.

Define $\varphi_{i}^{\prime}: S L(2, \mathrm{C}) \rightarrow G(V)$ by $\varphi_{i}^{\prime}=\pi \circ \varphi_{i}$ for each $i \in S$. Note that $\varphi_{i}^{\prime}$ is continuous for all $i \in S$.

If $G_{i}^{\prime}=\varphi_{i}^{\prime}(S L(2, \mathrm{C}))$, then for each $i \in S, G_{i}^{\prime}$ is either trivial, isomorphic to $S L(2, \mathrm{C})$, or isomorphic to $\operatorname{PSL}(2, \mathrm{C})$.

Theorem 1. Let $(V, d \pi)$ be an integrable representation for a Kac-Moody algebra g. If $G_{i}^{\prime}$ is nontrivial for some $i \in S$, then $G(A)$ is a universal covering group and a central extension of $G(V)$.

Proof. We have our continuous surjective homomorphism, $\pi: G(A) \rightarrow G(V)$. Since $G(A)$ is simply connected, we can prove that $G(A)$ is a universal cover by showing that $\pi$ is a local homeomorphism. Indeed, it suffices to show that $\pi$ is injective on some neighborhood of the identity.

Pick $i \in S$ so that $G_{i}^{\prime}$ is nontrivial. Since $G_{i}^{\prime} \cong S L(2, \mathrm{C})$ or $P S L(2, \mathrm{C})$, $\varphi_{i}^{\prime}=\pi \circ \varphi_{i}$ is a local homeomorphism. In particular, $\pi$ is injective on some neighborhood of 1 in $\varphi_{i}(S L(2, \mathbf{C})) \subseteq G(A)$. This shows that $G(A)$ is a universal cover of $G(V)$. By [10, $\S 7$, Example (c)], our proof is now complete.

Let $C(G)$ denote the center of a group $G$.

The next corollary strengthens Corollaries $4.4(a)$ and 4.5 of [5].

Corollary. If $(V, d \pi)$ is an integrable representation of a Kac-Moody algebra g, then $\operatorname{ker} \pi=G(A)$ or $\operatorname{ker} \pi \subseteq C(G(A))$.

Proof. If $G_{i}^{\prime}$ is trivial for all $i \in S$, then by definition of $\varphi_{i}^{\prime}$ and the fact that $G(A)$ is generated by $G_{i} S, \pi$ is trivial. In this case, $\operatorname{ker} \pi=G(A)$.

If $G_{i}^{\prime}$ is nontrivial for some $i \in S$, then Theorem 1 applies to give us $\operatorname{ker} \pi \subseteq C(G(A))$.

Assume now that $(V, d \pi)$ is an integrable representation of $\mathbf{g}$ with the property that $d \pi(x)$ is nonzero for some $x \notin \mathbf{h}$. Let $B(V)=\pi(B(A))$ and $N(V)=\pi(N(A))$. Since $\operatorname{ker} \pi \subseteq H(A)$, the following is immediate by Remark (2) in [1, Chapter IV, §2.1].

Theorem 2. $(G(V), B(V), N(V))$ is a group with $B N$-pair.

A building is a geometric configuration we associate to a group with $B N$-pair as follows. (Consult $[2,9]$ for the details.)

If $(G, B, N)$ is a group with $B N$-pair, then $W=B / N$ is a Coxeter group, generated by reflections $r_{i}$ for $i$ in some index set $I$. Any subgroup of $G$ which contains $B$ is called special. The special subgroups of $G$, it turns out, are 
precisely those of the form $B W^{\prime} B$ where $W^{\prime}$ is a subgroup of $W$ generated by reflections $r_{i}$ for $i \in I^{\prime} \subseteq I$. The $G$-conjugates of special subgroups are called the parabolic subgroups of $G$. The parabolics, partially ordered by opposite inclusion, comprise the building we associate to $(G, B, N)$.

The building we get from $(G(A), B(A), N(A))$ is the one we associate to $\mathbf{g}$.

Fix $(V, d \pi)$, an integrable representation of $\mathbf{g}$ such that $d \pi(x) \neq 0$ for some $x \notin \mathbf{h}$. Since $G(A)$ is a central extension of $G(V)$ and $C(G(A)) \subseteq$ $H(A) \subseteq B(A)$ (cf. [5]), the buildings associated to $(G(A), B(A), N(A))$ and $(G(V), B(V), N(V))$ are actually isomorphic. Note also that the Coxeter group associated to $(G(V), B(V), N(V))$ is isomorphic to $W$, the Weyl group associated to $\mathbf{g}$. In this case, the reflections which generate $B(V) / N(V)$ can be identified with the reflections in $\mathbf{h}^{*}$ determined by $\alpha_{i} \in \Pi$. We identify $W$ with $B(V) / N(V)$ in this setting so that if $V$ is a highest weight module with weight space $V_{\lambda}, w \cdot V_{\lambda}=V_{w(\lambda)}$ for all $w \in W$.

Buildings as inner ideal geometries. Now we assume that $\mathbf{g}$ is affine.

Let $(V, d \pi)$ be a faithful irreducible highest weight representation of $\mathbf{g}$. We define an inner ideal in $V$ as in [3]. By the inner ideal geometry associated to $(V, d \pi)$ we mean the inner ideals in $V$ partially ordered by opposite inclusion.

Suppose that $V$ has highest weight $\Lambda$. If $\lambda=\Lambda-\sum_{\alpha_{i} \in \Pi} k_{i} \alpha_{i}$ is a weight of $V$, the support of $\lambda, \operatorname{supp} \lambda$, is the set of simple roots $\alpha_{i}$ with $k_{i} \neq 0$. When $\Pi^{\prime} \subseteq \Pi$, we designate by $R\left(\Pi^{\prime}\right)$ the set of weights $\lambda$ with supp $\lambda \cap \Pi^{\prime}=\varnothing$.

The notion of a set of roots which is reduced $\bmod \Lambda$ is discussed in [3]. There we show that when $\Pi^{\prime} \subseteq \Pi$ is reduced $\bmod \Lambda, \mathscr{I}\left(\Pi^{\prime}\right)=\sum_{\lambda \in R\left(\Pi^{\prime}\right)} V_{\lambda}$ is an inner ideal. Every inner ideal in $V$, moreover, is a $G(V)$-translate of $\mathscr{I}\left(\Pi^{\prime}\right)$ for some reduced set of roots $\Pi^{\prime}$. When $\mathscr{I}$ is an inner ideal in $V$ and we write $\mathscr{I}=g \cdot \mathcal{I}\left(\Pi^{\prime}\right)$, it should be understood that $g$ is in $G(V)$ and $\Pi^{\prime} \subseteq \Pi$ is reduced $\bmod \Lambda$.

Let $\Pi_{\Lambda}=\left\{\alpha_{i} \in \Pi:\left(\Lambda, \alpha_{i}\right) \neq 0\right\}$. Assume that $\Pi=\Pi_{\Lambda}$ so that every subset of $\Pi$ is reduced $\bmod \Lambda$. Our next result says that the parabolic subgroups of $G(V)$ are the stabilizers of the inner ideals in $V$.

We designate the special subgroup $B(V)<r_{i}: i \notin S^{\prime}>B(V)$ by $P_{S^{\prime}}$.

Theorem 3. Let $\mathscr{I}=g \cdot \mathscr{I}\left(\Pi^{\prime}\right)$ be an inner ideal in $V$ with $\Pi^{\prime}=\left\{\alpha_{i} \in \Pi\right.$ : $\left.i \in S^{\prime}\right\}$. The stabilizer of $\mathscr{I}$ in $G(V)$ is $g P_{S^{\prime}} g^{-1}$.

Proof. Take $\lambda \in R\left(\Pi^{\prime}\right)$ so that $V_{\lambda} \subseteq \mathscr{I}\left(\Pi^{\prime}\right)$. For $b \in B(V), b \cdot V_{\lambda} \subseteq \sum_{\rho} V_{\rho}$ where $\operatorname{supp} \rho \subseteq \operatorname{supp} \lambda$. It follows that all such weights $\rho$ belong to $R\left(\Pi^{\prime}\right)$ thus that $B(V)$ stabilizes $\mathscr{I}\left(\Pi^{\prime}\right)$. Since any subgroup of $G(B)$ which contains $B(V)$ is special, it follows that the stabilizer of $\mathscr{I}\left(\Pi^{\prime}\right)$ in $G(V)$ is special.

Let $j$ belong to the complement of $S^{\prime}$ in $S$. Observe that for any $\lambda \in R\left(\Pi^{\prime}\right)$, $\operatorname{supp} r_{j}(\lambda) \cap S^{\prime}=\varnothing$. It follows that $r_{j}$ stabilizes $\mathscr{I}\left(\Pi^{\prime}\right)$. On the other hand, if $j$ belongs to $S^{\prime}$, then $\operatorname{supp} r_{j}(\Lambda) \cap S^{\prime}=\left\{\alpha_{j}\right\}$ since $\left(\Lambda, \alpha_{j}\right) \neq 0$. As $\Lambda \in R\left(\Pi^{\prime}\right)$ for all $\Pi^{\prime} \subseteq \Pi$, it follows that $r_{j}$ stabilizes $\mathscr{I}\left(\Pi^{\prime}\right)$ if and only if $j \notin S^{\prime}$. This gives us that $P_{S^{\prime}}$ is the stabilizer of $\mathscr{I}\left(\Pi^{\prime}\right)$.

Let $\mathscr{I}=g \cdot \mathscr{F}\left(\Pi^{\prime}\right)$ be an arbitrary inner ideal in $V$. It is clear that the elements in $g P_{S^{\prime}} g^{-1}$ stabilize $\mathscr{I}$. Suppose that $g^{\prime} \in G(V)$ stabilizes $\mathscr{I}$. We then have $g^{-1} g^{\prime} g$ stabilizing $\mathscr{I}\left(\Pi^{\prime}\right)$ so that $g^{-1} g^{\prime} g \in P_{S^{\prime}}$. This proves the theorem.

Our last result comes as a straightforward corollary to Theorem 3 . 
Corollary. The building associated to an affine Lie algebra can be realized as an inner ideal geometry.

Proof. Take $V$ to be an irreducible, integrable highest weight representation of g with highest weight $\Lambda$ satisfying $\Pi_{\Lambda}=\Pi$. Let $f$ map the inner ideals in $V$ to the parabolic subgroups of $G(V)$ by sending each inner ideal to its stabilizer. This is a well-defined function: if $g_{1} \cdot \mathscr{I}\left(\Pi_{1}\right)=g_{2} \cdot \mathscr{I}\left(\Pi_{2}\right)$, then Lemma 3.2 in [3] implies that $\Pi_{1}=\Pi_{2}$ so that $P_{S_{1}}=P_{S_{2}}$. From here we see that $g_{1}^{-1} g_{2}$ belongs to $P_{S_{1}}$ so that $g_{1} P_{S_{1}} g_{1}^{-1}=g_{2} P_{S_{2}} g_{2}^{-1}$.

We can also show that $f$ is bijective. Surjectivity is clear. By Theorem 2 in [2, Chapter V, $\S 2 \mathrm{~B}]$, distinct special subgroups cannot be conjugate. This implies that $f$ is injective.

Since the partial ordering on inner ideals and the partial ordering on parabolic subgroups are both defined as opposite inclusion of sets, $f$ is an isomorphism from the geometry of inner ideals in $V$ to the building determined by $\mathbf{g}$. The building associated to $\mathbf{g}$ can thus be realized as the inner ideal geometry associated to $(V, d \pi)$.

\section{REFERENCES}

1. N. Bourbaki, Groupes et algèbres de Lie, Chs. IV-VI, Hermann, Paris, 1968.

2. K. Brown, Buildings, Springer-Verlag, New York, 1989.

3. M. Dillon, Geometry and affine Lie algebras, J. Algebra 135 (1990), 96-111.

4. V. Kac, Infinite dimensional Lie algebras, Progr. Math., vol. 44, Birkhäuser, Boston, 1983.

5. V. G. Kac and D. H. Peterson, Defining relations of certain infinite-dimensional groups, Élie Cartan et les Mathématiques d'aujourd'hui, vol. hors série, Astérisque, 1985, pp. 165-208.

6. _ On geometric invariant theory for infinite-dimensional groups, Algebraic Groups, Utrecht 1986, Lecture Notes in Math., vol. 1271, Springer-Verlag, New York, 1987, pp. 109-142.

7. _ Regular functions on certain infinite-dimensional groups, Arithmetic and Geometry, Progr. Math., vol 36, Birkhäuser, Boston, 1983, pp. 141-166.

8. D. H. Peterson and V. G. Kac, Infinite flag varieties and conjugacy theorems, Proc. Nat. Acad. Sci. U.S.A. 80 (1983), 1778-1782.

9. M. Ronan, Lectures on buildings, Perspect. Math., vol. 7, Academic Press, San Diego, 1989.

10. R. Steinberg, Lecture notes on Chevalley groups, Yale University Lecture Notes, 1967.

Mathematics Department, Southern College of Technology, Marietta, Georgia 30060-2896

E-mail address: mdillonest 6000. sct.edu 